\title{
Ideología y Poder en el Arte del Manejo Antiguo del Agua
}

Bárbara W. Fash y Karla L.

Davis-Salazar

\section{Resumen}

Los templos y monumentos de Copán están repletos de símbolos del agua y de sustento, ambas fuerzas importantes en el desarrollo de complejidad social a través de la región y prevalente en toda Mesoamérica. Nuestro trabajo revela que los sistemas de agua manejados en Copán y áreas circundantes de Honduras tienen una historia larga y se manifiestan en diversas formas. Además de funciones prácticas, desde irrigación hasta el almacenamiento de agua, las fuentes y sistemas de agua tenían numerosos usos religiosos. Discutiremos cómo se concebía, usaba y se representaba el agua en tiempos antiguos y las formas que en que creemos se utilizaba su carácter sagrado en el arte para elevar la autoridad política. Por medio de nuestras investigaciones sabemos que la organización comunitaria era importante para la operación exitosa del manejo de los sistemas antiguos de agua. Puede que algunos de estos métodos sean relevantes en los paisajes actuales para mejorar las condiciones de vida, asegurar la potabilidad del agua y mejorar las cosechas.

Palabras Clave: Copán, símbolos del agua, manejo del agua, autoridad política, organización comunitaria.

\section{Abstract}

The temples and monuments of Copan are replete with symbols of water and sustenance, both important forces in the development of social complexity throughout the region and prevalent in Mesoamerica. Our work reveals that the water systems managed in Copan and neighboring regions of Honduras have a long history and they manifest in diverse forms. Besides their functional utilization, from irrigation to water storage, water sources and systems had numerous religious uses. We will discuss how water was conceived, used and represented in ancient times as well as we believe its sacred character was utilized in art to elevate political 
authority. From our research we know that communal organization was important to the successful management of ancient water systems. It might be that some of those methods are relevant to improve life conditions in current landscapes, to assure potable water and improve harvests.

Keywords: Copan, water symbols, water management, political authority, communal organization. .

Bárbara W. Fash, (bfash@fas.harvard.edu). Museo Peabody, Universidad de Harvard (Peabody Museum, Harvard University).

Karla L. Davis-Salazar, (karladavis@usf.edu) . Universidad del Sur de la Florida (South Florida University). 


\section{INTRODUCCIÓN}

¿Son el agua y el arte igual a poder? Como resultado de la investigación que presentamos aquí, parecería que los mayas antiguos construyeron comunidades fuertes con esta simple ecuación. También los rituales jugaron un papel importante en este desarrollo, aunque puede verse que por sí solos no eran suficientes para apaciguar a la deidad de la lluvia, Chaak. Lo crítico era saber cómo manejar el agua lluvia desde el momento que cae a la tierra lo que podría requerir un capital político significativo para realizarlo. De hecho, todas las ciudades mesoamericanas dependían fuertemente de fuentes seguras de agua, de alimentos y de sistemas de agua. Por toda América, muchas sociedades aborígenes se localizan alrededor de cuerpos de agua porque sus reuniones y actividades familiares dependen del agua. El agua se celebra como un componente básico para un sentido de comunidad, identidad, prácticas espirituales y la vida en general. Sin una entrega eficiente de estas necesidades básicas, el ambiente urbano no funcionará, se volverá insalubre, o aun más, dejará de existir. Hecho horrendo con el que nos familiarizamos mucho durante las inundaciones devastadoras del Huracán Mitch en Honduras y del Huracán Katrina en Nueva Orleans. En el mundo actual de llaves de agua, de irrigación intensiva para la agricultura y de piscinas, pareciera que hemos llegado a aceptar como realidades estos valiosos recursos y servicios. Los ocupantes urbanos de la antigua Mesoamérica quizá no lo tomaron así (Fash s.f.). Podemos quizá aprender del conocimiento pasado estudiando las tradiciones y prácticas del manejo antiguo del agua. Las lecciones del pasado pueden ayudarnos a mejorar las vidas de la gente hoy.

\section{IMAGINERÍA Y TECNOLOGÍA DEL AGUA}

Entender las dimensiones económicas y políticas del manejo del agua en la antigua Mesoamérica, demanda también la comprensión de las dimensiones mentales. Si revisamos el arte maya urbano, encontramos celebraciones de este recurso y el dominio de su uso. De hecho, encontramos que los ambientes urbanos utilizaron la naturaleza y el poder del agua, de las montañas, del maíz y del gobernante divino y sus contrapartes en el fuego y la sequía, para definir su paisaje urbano y su concepción de ciudad (Brady y Ashmore 1999; Dunning 1995, 2003; Dunning et al.2002; Fash 2005, s.f. Lucero 1999, 2005; Lucero y Fash 2006; Knapp y Ashmore1999; Puleston 1977; Schele y Mathews1998; Stark 1999; Taube 1996). 
La investigación sobre el antiguo manejo de las instalaciones de agua, sobre los patrones de asentamiento, sobre santuarios y sobre la imaginería relacionada con el agua, asociados tanto con el ambiente construido como con lugares sagrados nos permite valorar cómo la gente se integraba alrededor de este vital elemento de los mundos natural y social (Scarborough 1993, 1996, 1998, 2003). Al estudiar el manejo antiguo del agua por toda Mesoamérica, aprendemos no sólo acerca de las adaptaciones ecológicas y culturales a lo largo del espacio y del tiempo, sino que también aprendemos cómo el ritual estaba estrecha y universalmente ligado a su organización. Como un testimonio sobre su importancia, la veneración al agua y rituales comunitarios todavía se ven hoy día en las prácticas del agua en comunidades tradicionales o indígenas.

Al tomar una amplia perspectiva del manejo del agua en tiempos precolombinos a través del tiempo y del espacio, se pueden encontrar muchas modificaciones innovadoras del paisaje desde los olmecas del Formativo Temprano hasta los aztecas. Estas adaptaciones apuntan hacia un desarrollo temprano de complejidad política relacionada con el manejo del agua y con el control de parte de la élite del agua fresca. Muchos años de investigación en otras partes de Mesoamérica revelan que a través de la intensificación de la agricultura y de la modificación del paisaje, la élite gobernante estaba involucrada en el manejo del agua y en la producción de la agricultura como un medio para obtener poder político. El manejo azteca del agua en Tenochtitlán llegó a nuevos niveles con los campos elevados 0 "chinampas" y con las calzadas proveyendo un sistema masivo de ingeniería para sostener su imperio. Con la llegada de la colonización española, estos sistemas mesoamericanos se modificaron permanentemente (Carballal y Hernández 1989; Doolittle 1990).

En el Grupo Principal de Copán, donde las autoras realizaron investigaciones, el diseño multi-plaza de la Acrópolis proveyó un sistema tripartito de recolección que utilizaba el Patio Oriental, el Patio Occidental y el Patio de la Escalinata Jeroglífica. Es muy probable que las superficies lisas de los templos estucados canalizaran el agua hacia las plazas y, por medio de drenajes, la desviaban hacia el sur al estanque en el área urbana densamente poblada (Fash 2005, 2007; Fash y Davis-Salazar 2006). Aunque el sitio antiguo de Copán está ubicado a lo largo del río, durante la estación lluviosa los habitantes tempranos probablemente dependieron de estanques, manantiales naturales y aguadas. Hacia el noroeste del Grupo 9N-8, o Las Sepulturas, se encuentra una de las grandes depresiones rectangulares en el Valle. Karla Davis-Salazar (2003) investigó esta área y encontró 
evidencia para apoyar la idea de que fue un estanque antiguo. Una vez que el agua lluvia y el agua desviada se retenían en estanques, tanto las fuentes limpias de agua potable como los recursos acuáticos estarían continuamente disponibles durante todo el año. Numerosos investigadores han demostrado que las antiguas ciudades y complejos ceremoniales mesoamericanos pueden verse como réplicas arquitectónicas del paisaje sagrado. Las pirámides eran montañas que proveían un eje de comunicación con los dioses y los espíritus; las plazas que los circundaban eran los valles y las depresiones en donde se recaudaba el agua de distintos lado, surgiendo así estanques poco profundos. Los templos que en Copán se identifican por su iconografía como montañas sagradas fueron versiones tempranas del concepto náhuatl de altepetl, término para aldea o comunidad, el cual se traduce como "montaña de agua" o "montaña llena de agua". Por ejemplo, a una estructura del Período Clásico se le dio el nombre de campo "Índigo" y fue expresamente decorada con imaginería llamando la atención hacia sus funciones con respecto al agua. El agua sagrada que se recogía en su techo estucado se canalizaba hacia drenajes internos de la pirámide para que salieran por las bocas de una deidad terrestre y acuática. El mismo tema se expuso en la versión tardía que cubrió a Índigo, la muy conocida estructura del Templo 22, la cual está decorada con tuun witz, o deidades montañeses.

Existen tres elementos iconográficos que se encuentran por doquier en Copán y los que creemos representaban aspectos esenciales del manejo y rituales del agua en tiempos antiguos. Estos elementos incluyen: el tocado de nenúfares, el símbolo tuun escalonado y el medio nicho cuatrilobulado. Bárbara Fash (2005) sugiere que, cuando es llevado por gobernantes y nobles, el tocado de nenúfares explícitamente se relaciona a la estructura social de los deberes del manejo del agua y al papel ritual divino del agua de asegurar fertilidad y sustento. Como tal, puede que sea el tocado específico de los dueños regionales del agua.

Los motivos cuatrilobulados y medio cuatrilobulados se reconocen como representaciones de portales en general, más frecuentemente cuevas, así como también la apertura a hoyos de agua. También se les ha interpretado como representando nahb, o lugares de nenúfares (Houston et al. 2005; Schele y Grube 1990). Tanto montañas como toponimias a menudo se representan como medio cuatrilobulados_-por ejemplo los que se encuentran en Monte Albán y en Acanceh significando un ambiente frondoso (Miller 1991; Stuart 2000; Taube 2004; von Winning 1985). La evidencia de Copán sugiere que también se utilizaban para 
designar lugares con nombres y posiblemente santuarios en circuitos rituales (Fash 2005). Tal como lo ha demostrado Karen Bassie (1996), las gotas de agua en una cueva se consideran extremadamente sagradas y puras. Aún hoy día se recogen para la preparación de bebidas rituales y para pociones curativas. El símbolo para estas gotas de agua es el signo tuun, que adorna las cabezas de los witz o las deidades montañeses que se encuentran tanto en las fachadas como en las estelas de Copán. Aquí se puede ver el ejemplo sacado de la fachada de la Estructura 10L41, en el conjunto residencial real, donde un signo tuun aparece dentro de una forma escalonada. Una vasija de entierro también asociada con esta estructura fue pintada con un diseño negro mostrando dos motivos similares escalonados, colocados uno sobre el otro, y gotitas cayendo entre ellos (Fash 2005). En el corpus de la escultura del Clásico Tardío de Copán, estos elementos relacionados con el agua se representaban continuamente, significando la importancia de los lugares de agua. Ciertamente, el nombre Tollán o "lugar de las cañas" (Stuart 2000), que estaba en el corazón de la fundación de ciudades sagradas por toda Mesoamérica es descriptiva de una laguna o un ambiente acuático, indicando el poderoso papel del agua en el desarrollo de la civilización en Mesoamérica. Los estudios sobre la subsistencia maya de Robert Rands (1955) y de Dennis Puleston $(1976,1977)$ llamaron la atención a la iconografía de flora y de fauna acuáticas y al simbolismo del agua. Ellos asociaron estos motivos con ambientes perezosos de arroyos, ríos, lagunas, aguadas, pantanos y planicies inundables de ríos, mencionando específicamente los motivos de cocodrilos y nenúfares.

\section{El Altar T como Evidencia de una Tecnología de Agua Avanzada}

Los sitios mesoamericanos generalmente se diseñaron en cuatro cuadrantes, en conexión con las direcciones cardinales. Consideramos que había cuatro posiciones significativas direccionales en la ciudad antigua copaneca que pudieron haber sido áreas de santuarios. Agua, cavernas o simples aperturas en la tierra parecen jugar un papel en la selección de estos locales sagrados y de estos santuarios antiguos. Cerca de La Laguna, en la orilla sur del valle y cerca de la Estela 12 en la orilla este, durante visitas antiguas rituales, los antiguos dejaron ofrendas de jade, cerámica y fragmentos de escultura en aperturas y en rasgos naturales (Núñez Chinchilla 1972). La Estela 10 y su caverna al oeste y la Estela 12 al este pudieron haber sido marcadores para santuarios direccionales en sus respectivas cimas de montaña; ambas asociadas con cavernas y fuentes de agua. La Laguna al sur pudo haber sido el área de santuario al sur del valle. Un santuario al norte estaba quizá en la 
cima del Cerro de Las Mesas, local marcado hoy día por una cruz y el cual es el punto para la ceremonia del 3 de mayo, Día de la Cruz (Fash s.f.).

\section{Implicaciones Sociales y Políticas del Manejo del Agua}

El mensaje con respecto al papel preponderante que jugó el agua dentro de la sociedad antigua maya se dio a conocer en las fachadas esculpidas de los sectores residenciales grandes de Copán que aparecen a lo largo y ancho del valle. En Las Sepulturas, al este del Grupo Principal, el edificio dominante, la Estructura $9 \mathrm{~N}-82$, estaba adornada con una escultura de fachada con una figura central que llevaba un tocado de nenúfar flanqueada por dos figuras sentadas llevando tocados de maíz. Nosotros utilizamos el modelo etnográfico descrito por Vogt para Zinacantán con el objetivo de interpretar las figuras que aparecen con tocados de maíz, como representativas de grupos de linajes basados en la agricultura (Fash 2005; Fash y Davis-Salazar 2006). La figura central más importante que lleva el tocado de nenúfar puede que represente la cabeza o líder del grupo de la cavidad de agua quien desempeñaba deberes similares a los de los administradores modernos zinacantecos. Los grupos de linaje de Copán basados en la tierra quizá se unían para formar grupos más grandes de cavidades de agua, similares a los grupos zinacantecos de cavidades de agua. Estas unidades sociales más grandes, inclusivas de grupos agrícolas, quizá se unían como resultado del uso común de una fuente de agua o como resultado de la administración de un sistema urbano, como un estanque. Un grupo de cavidad de agua habría reforzado límites territoriales por medio de la participación del grupo en un sistema de creencias que requería ofrendas ceremoniales a los espíritus y a los patrones ancestrales de la cavidad de agua, junto con el mantenimiento de la cavidad de agua y de santuarios relacionados. A pesar de que la población era diversificada y cambiante, el manejo de las aguas dentro de comunidades locales era una fuerza potencial de cohesión, creando y dándole poder a una identidad local.

En el área de la residencia real de Copán, El Cementerio, hacia el sur del grupo principal, las figuras en la estructura central aparecen todas llevando tocados de nenúfar. Esto nos sugiere que los nobles de la realeza en este grupo eran los amos regionales del agua y tenían jurisdicción sobre el estanque en el área cercana de El Bosque y también sobre la ingeniería del precinto sagrado. Quizá la dinastía gobernante de Copán era el grupo corporativo más grande de administradores del agua, dentro de una organización compleja, unida por actividades del manejo 
de agua, que pudo haber tenido un papel preponderante en el gobierno central de la ciudad durante los siglos siete y ocho. El colapso de la élite gobernante en el año 822 d.C. muy posiblemente apuntaba a la desintegración eventual de los poderosamente manejados sistemas de agua. Éste es un modelo que también lo propone Lucero para el área maya en general (2006).

Los asentamientos residenciales a lo largo y ancho del Valle de Copán pudieron haber incorporado dos o más grupos de cavidad de agua para formar aún conjuntos más grandes, definiendo aldeas o lo que se llaman sian otot entre los maya chortí (Fash 1983). El modelo de Bárbara Fash, en cuanto a la organización socio política antigua dentro del Valle de Copán centrado en rasgos acuáticos, también propone que los grupos sian otot enviaban un representante 0 perito regional de agua a una casa de concejo central, o popol nah. Ella sugiere que los medio nichos cuatrilobulados con íconos interiores que se encuentran en fachadas residenciales particulares puede que encierren el topónimo relacionado con el agua o el patrón ancestral del grupo y señalar la residencia del representante. En Copán, la Estructura 22A en el Grupo Principal, la que se ha interpretado como el popol nah central (Fash y otros 1992), se cree que el glifo de pescado en frente es el topónimo para el área residencial real, El Cementerio. Una escultura de pescado en un escondite se encontró dentro de la Estructura 32, la estructura central de ese grupo (Andrews y Fash 1992). Modelos similares existieron en otros sitios como Xochicalco, México, donde topónimos de agua aparecen prominentemente en la estructura más decorada del sitio (Wilkerson 1999).

Las numerosas representaciones de los medio nichos cuatrilobulados sirven como ejemplos de la imaginería cueva/cavidad de agua que proponemos estaba en el corazón del sistema de creencias y de la jerarquía política para los cuales el gobernante era la figura central. El concejo que se reunía para regular los aspectos sociales y políticos de las comunidades del valle que componían el reino posiblemente tomaron forma cuando K'inich Yax K'uk' Mo', el primer gobernante dinástico, centró la ciudad propiamente y comenzó la configuración de la plaza tríada (o nahb) y que se convirtió en el Grupo Principal (ver a Sharer y Traxler 1992 para conocer sobre la arquitectura temprana de la Acrópolis). La ingeniería del agua se convirtió en una función importante con el fin de asegurar un recurso vital para las crecientes comunidades del estado, mientras que los gobernantes y los linajes invocaban a sus dioses ancestrales en la geografía sagrada para legitimar su poder y para unificar a las comunidades locales. Medios nichos cuatrilobulados similares con personajes humanos que aparecen en la tapadera del sarcófago de Pacal en Palenque, tocados de nenúfar por nobles en los murales de Bonampak y 
una combinación de estos motivos en vasijas de Tikal nos llevan a especular si no habría en existencia un modelo similar en aquellos sitios y en otros del área maya.

Nueva evidencia de fuera y cerca del área maya parece sugerir que quizá sus vecinos, los lencas, siguieron un modelo similar en el manejo del agua. A unos pocos kilómetros de Copán, en el Valle de Palmarejo en el noroeste de Honduras, pueblos y aldeas antiguas lencas participaron en redes de comercio a larga distancia con ciudades-estado mayas más grandes, incluyendo a Copán. Los patrones de asentamiento prehispánico en Palmarejo sugieren que familias viviendo a lo largo de quebradas (riachuelos estacionales) se organizaban en grupos de uso de recursos, o "comunidades de quebrada", para manejar recursos ecológicos clave, incluyendo el agua. Y, así como en Copán, en Palmarejo, el centro político más grande del Valle de Palmarejo, el agua se encausaba hacia el centro y se recolectaba en un estanque grande, muy seguramente sirviendo tanto para funciones prácticas como religiosas de la élite. Estos nuevos hallazgos, sumados a la evidencia de Copán, sugieren que la organización comunitaria para la recolección y control del agua con fines domésticos (incluyendo rituales) parece haber sido un sistema común de manejo utilizado por los antiguos mesoamericanos, mientras que un control del manejo de agua más centralizado por la élite, como los manejadores de agua propuestos para Copán y para los líderes lencas de Palmarejo, se acomodaba mejor para fines políticos más amplios. Esta interpretación sugerida tiene implicaciones para el manejo de agua contemporáneo.

\section{Tradiciones Continuas y Aplicaciones Prácticas}

Los grupos indígenas contemporáneos continúan aferrándose con tenacidad a las ceremonias de cuevas y de agua en un empeño por sostener sus creencias religiosas, unificar comunidades alrededor de metas económicas comunes y para asegurar la constante disponibilidad de agua. Los rituales y festivales, que numerosas comunidades contemporáneas por toda Mesoamérica mantienen como una relación con manantiales sagrados y con los sistemas de captación de agua, ayudan a salvar la pureza de los recursos comunitarios, a controlar derechos de agua y a proteger los ambientes sagrados. No obstante, el número menguante de comunidades indígenas, la falta de apoyo de los gobiernos centrales, la falta de iniciativas corporativas de comercialización y las poblaciones florecientes alrededor de ciudades grandes amenazan estos sistemas. Puede que la comprensión de las profundas raíces históricas de las prácticas exitosas de agua que han sobrevivido desde tiempos antiguos ayude a conservar la cohesión de la comunidad entre 
grupos contemporáneos, al mismo tiempo que agencias de desarrollo y otros grupos trabajan para traer agua potable limpia y segura a aquellos quienes no la tienen.

Se ha argumentado que la perspectiva a largo plazo de la arqueología es una ventaja única de esta disciplina para comprender las relaciones contemporáneas humano-ambiente. Sin embargo, con unas pocas excepciones notables (e.g., el restablecimiento que Clark Erickson ha hecho de la agricultura en campos alzados en partes de la América del Sur), rara vez se incorporan datos arqueológicos sobre tradiciones y prácticas pasadas en las iniciativas de desarrollo que se enfocan en aspectos ambientales. Lo que sugiere la evidencia arqueológica que aquí se presenta es la necesidad de considerar métodos alternos, quizá más eficientes y efectivos de utilización, control y conservación del agua de los que actualmente se utilizan en contextos modernos. El estudio de la interacción humana, cómo se utilizaron y se mantuvieron los sistemas hidráulicos antiguos y los factores que llevaron al colapso de estos sistemas puede proveer lecciones para las políticas modernas globales de agua como también puede proveer soluciones prácticas en cuanto a las situaciones de la actual carestía de agua.

El manejo aparente del agua por la élite antigua gobernante como una fuente de poder político ilumina nuestras preocupaciones contemporáneas en cuanto al corretaje corporativo del agua por la élite. Con base a los sistemas antiguos, se puede concluir que, aunque las políticas del agua requieren la mediación de las autoridades o concejos, el control estricto corporativo/élite al final caerá con los cambios sociopolíticos y que las prácticas tradicionales de veneración del agua, que están todavía en uso, sugieren que comunidades pequeñas en vez de centros grandes pueden ser una clave importante para la eficiencia y el sostenimiento de los proyectos de la conservación del agua en el mundo actual. 


\section{BIBLIOGRAFÍA}

- Andrews V., Wyllys E., Fash B., Fash W. (1992). Continuity and Change in a Royal Maya Residential Complex at Copan . Ancient Mesoamerica, 63-88.

- Bassiet-Sweet, K. (1996). At the Edge of The World: Caves and Late Classic Maya World View. Norman: University of Oklahoma Press.

- Baudez, C. (1994). Maya Scullpture of Copan: The Iconography. Norman, London: University of Oklahoma Press.

- Brady J., Ashmore W. (1999). Mountains, Caves, Water: Ideational Landscapes of the Ancient Maya. En W. Ashmore y A. Bernard Knapp, Archaeologies of Landscape: Comtemporary Perspectives (págs. 124-145). London: Blackwell.

- Carballal S., Hernandez M., Hernandez M. . (1989). Las calzadas prehispánicas de la Isla de México:algunas consideraciones acerca de sus funciones. Arqueología, 71-80.

- Davis-Salazar, K. (2003). Late Classic Maya Water Management and Community Organization at Copan, Honduras. Latin American Antiquity, 275-299.

- Doolitle, W. (1990). Canal irrigation in Prehistoric Mexico: The Sequence of Technological Change. Austin: University of Texas Press.

- Dunning, N. . (1995). Coming Together at the Temple Mountain: Enviroment, Subsistence, and the Emergence of Classic Maya Segmentary States. En N. Grube, Emergence of Classic Maya Civilization, Acta Mesoamericana No. 8 (págs. 61-70). Mockmuhl: Verlag von Flemming.

- Dunning, N. (2003). Birth and Death of Waters: Enviromental Change, Adaptation, and Symbolism in the Southern Maya Lowlands. En A. Breton, A. M. Becquelin, M.H. Ruiz, Espacios Mayas: Usos, Representaciones, Creencias (págs. 49-76). México: Universidad Autónoma de México .

- Dunning N., Luzzadder-Beach S., Beach T., Jones J., Scarborough V.L., Culbert P. (2002). Arising from the Bajos: Anthropogenic Change of Wetlands and the Rise of Maya Civilization. Annals of the Association of American Geographers, 267-283.

- Fash, B. (1992). Late Classic Architectural Sculpture Themes in Copán . Ancient Mesoamerica, 89-104. 
- Fash, B. (2005). Iconographic Evidence for Water Management at Copán, Honduras. En E. W. Andrews, W.L. Fash, Copán: History of an Ancient Maya Kingdom (págs. 103-138). Santa Fe: School of American Research Press.

- Fash, B. (S.F.). Waterly Places and Urban Foundations Depicted in Maya Art and Architecture. Dumbarton Oaks Conference The Art of Urbanism. Ciudad de México, Octubre 2006.

- $\quad$ Fash B., Fash W., Larios R., Lane S., Schele L., Stomper J., Stuart D. (1991). Investigations of a Maya Council House from Copán, Honduras. Journal of Field Archaeology, 419-442.

- Fash B., Davis-Salazar K.L. (2006). Copan Water Ritual and Management: Imagery and Sacred Place. En L.J. Lucero, B.W. Fash, Precolumbian Water Management: Ideology, Ritual and Power (págs. 129-143). Tucson: University of Arizona Press.

- Fash, W. L. (1983). Deducing Social Organization from Classic Maya Settlement Patterns: A Case Study from the Copán Valley. En R. Leventhal, A. Kolata, Civilization in the Ancient Americas, Essays in Honor of Gordon R. Willey (págs. 261-288). Albuquerque: University of New Mexico Press, Peabody Museum of Archaeology and Ethnology .

- Lucero, L. J. (2006). The Rise and Fall of Classic Maya Rulers . Austin: University of Texas Press.

- Lucero L.J., Fash B.W. (eds). (2006). Precolumbian Water Management: Ideology, Ritual and Power. Tucson: University of Arizona Press.

- Miller, V. (1991). The Frieze of the Palace of Stuccoes, Acanceh, Yucatan, Mexico. Washington : Dumbarton Oaks.

- Puleston, D. E. (1977). Art and Archaeology of Hydraulic Agriculture in the Maya Lowlands. En N. Hammond, Social Process in Maya Prehistory, Studies in the Memory of Si Eric Thompson (págs. 449-467). London: Academic Press.

- Rands, R. L. (1955). Some Manifestations of Water in Mesoamerican Art. Smithsonian Bureau of american Ethnology, Anthropological Papers No. 48, 265-393.

- Scarborough, V. (1993). Water Management in the Southern Maya Lowlands: An Accretive Model for the Engineered Landscape. EnV.L Scarboroug, B.L. 
Isaac, Economic Aspects of Water Management in the Prehispanic New World (págs. 17-69). Greenwich: Jai Press Inc.

- Scarborough, V. (1996). Reservoirs and Watersheds in the Central Maya Lowlands. En S. L. Fedick, The Managed Mosaic: Ancient Maya Agriculture and Resource Use (págs. 304-314). Salt Lake City: University of Utah Press.

- Scarborough, V. (1998). Ecology and Ritual: Water Management and the Maya. Latin American Antiquity, 135-159.

- Scarborough, V. (2003). The Flow of Power: Ancient Water Systems and Landscapes. Santa Fe: School of American Research Press.

- Schele L., Grube N. (1990). The Glyph for Plaza or Court . Copan Acropolis Archaeological Project, Instituto Hondureño de Antropología e Historia .

- Schele L., Mathews P. (1998). The Code of Kings. New York: Simon and Schuster.

- $\quad$ Sharer R. J., Miller J.,Traxler L. (1992). Evolution of Classic Period Architecture in the Eastern Acropolis, Copán: A Progress Report . Ancient Mesoamerica, 145-160 .

- Stark, B. (1999). Commentary: Ritual, Social Identity, and Cosmology: Hard Stones and Flowing Water. En D. C. Grove, R.M. Joyce, Social Patterns n PreClassic Mesoamerica (págs. 301-317). Washington: Dumbarton Oaks.

- Stuart, D. (2000). The Arrival of Strangers: Teotihuacan and Tollan in Classic Maya History . En D. Carrasco,L. Jones, S. Sessions, Mesoamerica's Classic Heritage: From Teotihuacan to the Aztecs (págs. 465-513). Boulder: University of Colorado Press.

- Taube, K. A. (1996). The Olmec Maize God. RES 29/30, 39-81.

- Taube, K. A. (2004). Structure 10L-16 and its Early Antecedents: Fire and Evocation and Resurrection of K'inich Yax K'uk'Mo'. En E. Bell, M. Canuto, R. Sharer, Understanding Early Classic Copan (págs. 265-295). Philadelphia: University of Pennsylvania Press .

- Vogt, E. Z. (1969). Zinacantan: A Maya Community in the Highlands of Chiapas. Cambridge: Harvard University Press. 
- Von Winning, H. (1985). Two Maya Monuments in Yucatan: The Palace of the Stuccoes at Acanceh and the Temple of the Owls at Chichen Itzá. Los Angeles: Southwest Museum .

- Wilkerson, J. K. (1999). Public Buldings and Civic Spaces at Xochicalco, Morelos . En J. K. Kowalski, Mesoamerican Architecture as Cultural Symbol. New York : Oxford University Press. 